\title{
PERTURBATIONS OF SEMI-FREDHOLM OPERATORS BY OPERATORS CONVERGING TO ZERO COMPACTLY
}

\author{
SEYMOUR GOLDBERG
}

ABSTRACT. Let $\left\{K_{n}\right\}$ be a sequence of bounded linear operators mapping a Banach space $X$ into a Banach space such that $K_{n} \rightarrow 0$ strongly and $\left\{K_{n} x_{n}\right\}$ is relatively compact for every bounded sequence $\left\{x_{n}\right\} \subset X$; e.g., $\left\|K_{n}\right\| \rightarrow 0$. Given $T$ a semi-Fredholm operator, it is shown that for all sufficiently large $n, T+K_{n}$ has nullity and deficiency not exceeding that of $T$ while the index of $T+K_{n}$ equals that of $T$. Properties of the minimum modulus of $T+K_{n}$ are al so given.

Let $X$ and $Y$ be Banach spaces. A sequence $\left\{K_{n}\right\}$ of bounded linear operators mapping $X$ into $Y$ is said to converge to zero compactly, written $K_{n} \stackrel{c}{\rightarrow} 0$, if

(1) $K_{n} x \rightarrow 0$ for all $x \in X$;

(2) $\left\{K_{n} x_{n}\right\}$ is relatively compact for every bounded sequence $\left\{x_{n}\right\} \subset X$. Clearly, $\left\|K_{n}\right\| \rightarrow 0$ implies $K_{n} \stackrel{c}{\rightarrow}$. 0 . If $\bigcup_{n} K_{n} S$ is relatively compact, where $S$ is the 1 -ball in $X$, then (2) is satisfied. In this case $\left\{K_{n}\right\}$ is called collectively compact and was intensively studied in [1].

In this paper we present properties of the nullity, deficiency and index of operators of the form $T+K_{n}$, where $T$ is a semi-Fredholm operator and $K_{n} \stackrel{c}{\rightarrow} 0$. We obtain theore ms analogous to those given in [3] and [41, where the requirement was that $K_{n}$ be "small enough" in norm. We generalize the results and simplify the proofs appearing in [5] where $X$ and $Y$ were assumed to be Hilbert spaces with $T$ a bounded semi-Fredholm operator.

Throughout this paper $T$ is ass umed to be a closed linear operator with domain $\mathcal{D}(T) \subset X$ and range $R(T)$ a closed subspace of $Y$.

$\alpha(T)=\operatorname{dim} \Re(T)$, where $\Re(T)$ is the kernel of $T$ and $\beta(T)=$ co $\operatorname{dim} R(T)$. If, in addition, $\alpha(T)$ or $\beta(T)$ is finite, $T$ is called a semi-

Received by the editors May 21, 1973.

AMS (MOS) subject classifications (1970). Primary 47 A55, 47B30.

Key words and phrases. Semi-Fredholm, index, nullity, deficiency, minimum modulus. 
Fredholm operator with index $\kappa(T)=\alpha(T)-\beta(T)$. If both $\alpha(T)$ and $\beta(T)$ are finite, $T$ is called a Fredholm operator.

For properties of semi-Fredholm operators the reader is referred to [3] and [4].

Preliminary remarks.

I. $R(T)$ is closed if and only if $\gamma(T)=\inf \|T x\| / d(x, \Re(T))=\left\|\hat{T}^{-1}\right\|^{-1}$ $>0$, where $d(x, \Re(T))$ is the distance from $x$ to $\Re(T)$ and $\hat{T}$ is the $1-1$ operator induced by $T$.

II. If $\left\{A_{n}\right\}$, a sequence of bounded linear operators on $X$ with range in $Y$, converges strongly to zero, then $\left\{\left\|A_{n}\right\|\right\}$ is bounded and $\left\{A_{n}\right\}$ con verges to zero uniformly on totally bounded sets.

III. If $\left\{y_{n}\right\}$ is a bounded sequence in $R(T)$, then there exists a bounded sequence $\left\{x_{n}\right\}$ such that $T x_{n}=y_{n}$.

This follows readily from $y_{n}=T v_{n}$ and $\left\|y_{n}\right\|=\left\|T v_{n}\right\| \geq \gamma(T) d\left(v_{n}, \gamma(T)\right)$.

IV. If $\alpha(T)<\infty$ and $\left\{x_{n}\right\}$ is a bounded sequence such that $\left\{T x_{n}\right\}$ converges, then $\left\{x_{n}\right\}$ has a convergent subsequence; for, since $R(T)$ is closed, $T x_{n} \rightarrow T x$ and therefore $x_{n}+\Re(T)=\hat{T}^{-1} T x_{n} \rightarrow \hat{T}^{-1} T x=x+\Re(T)$ in $x / \Re(T)$. Thus there exists $z_{n} \in \Re(T)$ such that $x_{n}+z_{n} \rightarrow x$. Since $\left\{z_{n}\right\}$ is bounded in finite dimensional space $\Re(T)$, it, and therefore $\left\{x_{n}\right\}$, has a convergent subsequence.

Basic Lemma [2, p. 190]. If $M$ and $N$ are subspaces of $X$ and $\operatorname{dim} M>\operatorname{dim} N$, there exists an $m \in M$ such that $1=\|m\|=d(m, N)$.

Throughout the remainder of this paper, $K_{n} \stackrel{c}{\rightarrow} 0$.

Lemma 1. If $\alpha(T)<\infty$ and $\boldsymbol{r}(T)$ is complemented in $X$ by a closed subspace $M$, then there exists $a p$ and $c .>0$ such that for $n \geq p, T_{M}+K_{n}$ is 1-1 and $\gamma\left(T_{M}+K_{n}\right) \geq c$, where $T_{M}$ is the restriction of $T$ to $M \cap \mathfrak{D}(T)$.

Proof. Suppose $\left\{\gamma\left(T_{M}+K_{n}\right)\right\}$ has a subsequence converging to zero. For simplicity, let $\gamma\left(T_{M}+K_{n}\right) \rightarrow 0$. There exists $\left\{m_{n}\right\} \subset M$ such that $\left\|m_{n}\right\|=1$ and $\left(T+K_{n}\right) m_{n} \rightarrow 0$. Since $K_{n} \stackrel{c}{\rightarrow} 0,\left\{K_{n} m_{n}\right\}$, and therefore $\left\{T m_{n}\right\}$, has a convergent subsequence. Thus by IV, $\left\{m_{n}\right\}$ has a convergent subsequence and by II, $\left\{K_{n} m_{n}\right\}$, and therefore $\left\{T m_{n}\right\}$, has a subsequence converging to zero. This is impossible since $T_{M}$ has a bounded inverse. This argument also shows that $T_{M}+K_{n}$ is 1-1 for sufficiently large $n$; otherwise, a sequence $\left\{m_{n}\right\}$ with the above properties would obviously exist which leads to a contradiction. 
The assumption $K_{n} \stackrel{c}{\rightarrow} 0$ does not even imply that the sequence $\left\{K_{n}^{\prime}\right\}$ of conjugate operators converges strongly. The following simple example taken from [1] confirms this.

Take $X=Y=l_{2}$. Define $K_{n} x=x_{n} e_{1}$, where $x=\Sigma_{1}^{\infty} x_{i} e_{i},\left\{e_{i}\right\}$ the usual set of unit vectors. Then $K_{n}^{\prime} e_{1}=e_{n}$ does not converge in $l_{2}$.

In light of this example, it is somewhat surpris ing that the following "dual" lemma holds.

Lemma 2. Let $\mathfrak{D}(T)$ be dense in $X$. If $\mathfrak{R}(T)$ is complemented in $Y$ by a closed subspace $W$, then there exists a $p$ and $c>0$ such that for $n \geq p, T^{\prime}+K_{n}^{\prime}$ is $1-1$ on $W^{\circ}=\left\{y^{\prime} \in Y^{\prime}: y^{\prime} W=0\right\}$ and $\gamma\left(T_{0}^{\prime}+K_{n}^{\prime}\right) \geq c$, where $T_{0}^{\prime}$ is the restriction of $T^{\prime}$ to $W^{\circ} \cap \mathfrak{D}\left(T^{\prime}\right)$.

Proof. $Y=R(T) \oplus W$ and $Y^{\prime}=\mathfrak{R}(T)^{\circ} \oplus W^{\circ}$. Suppose $\gamma\left(T_{0}^{\prime}+K_{n}^{\prime}\right)$ has a subsequence converging to zero. For simplicity, let $\gamma\left(T_{0}^{\prime}+K_{n}^{\prime}\right)$ $\rightarrow 0$. There exists $\left\{y_{n}^{\prime}\right\} \subset W^{\circ}$ such that $1=\left\|y_{n}^{\prime}\right\|$ and $\left(T^{\prime}+K_{n}^{\prime}\right) y_{n}^{\prime} \rightarrow 0$. Choose $y_{n}$ so that $1=\left\|y_{n}\right\|$ and $y_{n}^{\prime} y_{n} \geq 1 / 2$. Now $y_{n}=T v_{n}+w_{n}, w_{n} \in W$ and $\left\{T v_{n}\right\}$ is bounded since $R(T)$ is closed and complemented by $W$. Hence by III, there exists a bounded sequence $\left\{x_{n}\right\}$ such that $T x_{n}=T v_{n}$. Furthermore, $y_{n}^{\prime} v \rightarrow 0$ for all $v \in Y$. To see this, $y_{n}^{\prime} T x=\left(T^{\prime}+K_{n}^{\prime}\right) y_{n}^{\prime}(x)$ $-y_{n}^{\prime} K_{n} x \rightarrow 0$. Since $y_{n}^{\prime}$ is in $W^{\circ}$ and $R(T)$ is complemented by $W$, $y_{n}^{\prime} v \rightarrow 0$ for all $v \in Y$. Now

$$
1 / 2 \leq y_{n}^{\prime} y_{n}=\left(T^{\prime}+K_{n}^{\prime}\right) y_{n}^{\prime}\left(x_{n}\right)-y_{n}^{\prime} K_{n} x_{n} .
$$

Since $\left\{x_{n}\right\}$ is bounded, $\left\{K_{n} x_{n}\right\}$ is totally bounded which, together with the observation that $y_{n}^{\prime} v \rightarrow$ J for all $v \in Y$; implies that $\left\{y_{n}^{\prime} K_{n} x_{n}\right\}$ converges to zero. Therefore $(*)$ cannot hold since $\left(T^{\prime}+K_{n}^{\prime}\right) y_{n}^{\prime} \rightarrow 0$.

The above argument also shows that $T^{\prime}+K_{n}^{\prime}$ is $1-1$ on $W^{\circ}$ for all sufficiently large $n$; otherwise a sequence $\left\{y_{n}^{\prime}\right\}$ with the above properties would obviously exist which leads to a contradiction.

Theorem 1. Suppose $a(T)<\infty$. There exists a $p$ such that:

(1) $T+K_{n}$ has a closed range and $\alpha\left(T+K_{n}\right) \leq \alpha(T), n \geq p$.

(2) $\alpha\left(T+K_{n}\right)=\alpha(T), n \geq p$, if and only if $\inf _{n \geq p} \gamma\left(T+K_{n}\right)>0$. In this case, $X=M \oplus \Re\left(T+K_{n}\right), n \geq p$, where $\Re(T)$ is complemented by the closed subspace $M$.

Proof. $X=M \oplus \Re(T)$ for some closed subspace $M$. Let $p$ and $c>0$ be as in Lemma 1 and $n \geq p$. Then $\left(T+K_{n}\right) M$ is closed by $I$ and the finite dimensionality of $\Re(T)$ implies $R\left(T+K_{n}\right)=\left(T+K_{n}\right) M+K_{n} \Re(T)$ is 
closed. Moreover, by Lemma 1, $M \cap \Re\left(T+K_{n}\right)=\{0\}$. Hence $X=M \oplus$ $\boldsymbol{r}(T) \supset M \oplus \Re\left(T+K_{n}\right)$ which implies $\alpha\left(T+K_{n}\right) \leq \alpha(T)$.

Suppose $\alpha\left(T+K_{n}\right)=\alpha(T), n \geq p$. Then $X=M \oplus \Re\left(T+K_{n}\right)$ and for $x=m_{n}+z_{n}, m_{n} \in M, z_{n} \in \mathfrak{l}\left(T+K_{n}\right)$, we have by Lemma 1 that

$$
\begin{aligned}
\left\|\left(T+K_{n}\right) x\right\| & =\left\|\left(T+K_{n}\right) m_{n}\right\| \geq c\left\|m_{n}\right\| \\
& \geq c d\left(m_{n}, \Re\left(T+K_{n}\right)\right)=c d\left(x, \Re\left(T+K_{n}\right)\right) .
\end{aligned}
$$

Thus $\gamma\left(T+K_{n}\right) \geq c>0, n \geq p$. Conversely, suppose $\gamma\left(T+K_{n}\right) \geq c>0$, $n \geq p$, but that $\alpha\left(T+K_{n}\right) \neq \alpha(T)$. Then, from (1), $\alpha\left(T+K_{n}\right)<\alpha(T)$. By the basic lemma there exists $\left\{z_{n}\right\} \subset \Re(T)$ such that $1=\left\|z_{n}\right\|=d\left(z_{n}, \Re\left(T+K_{n}\right)\right)$. Hence for $n \geq p$,

$$
0<c=c d\left(z_{n}, \Re\left(T+K_{n}\right)\right) \leq\left\|\left(T+K_{n}\right) z_{n}\right\|=\left\|K_{n} z_{n}\right\| .
$$

Since $r(T)$ is finite dimensional, $\left\{z_{n}\right\}$ has a convergent subsequence and therefore by II, $\left\{K_{n} z_{n}\right\}$ has a subsequence converging to zero, contradicting $(*)$.

Theorem 2. If $R(T)$ is complemented in $Y$ (by a closed subspace) and $T$ is densely defined, there exists a $p$ such that for $n \geq p, a\left(T^{\prime}+K_{n}^{\prime}\right)$ $\leq \alpha\left(T^{\prime}\right)$.

If $\beta(T)<\infty$, there exists a $p$ such that:

(i) $T+K_{n}$ has closed range with $\beta\left(T+K_{n}\right) \leq \beta(T), n \geq p$.

(ii) $\beta\left(T+K_{n}\right)=\beta(T), n \geq p$, implies inf ${ }_{n \geq p} \gamma\left(T+K_{n}\right)>0$.

Proof. Let $W$ and $p$ be chosen as in Lemma 2 with $n \geq p$. Then $Y^{\prime}=R(T)^{\circ} \oplus W^{\circ}=\Re\left(T^{\prime}\right) \oplus W^{\circ}$. Since $T^{\prime}+K_{n}^{\prime}$ is $1-1$ on $W^{\circ}, Y^{\prime} \supset$ $\mathfrak{r}\left(T^{\prime}+K_{n}^{\prime}\right) \oplus W^{\circ}$. Thus $\alpha\left(T^{\prime}+K_{n}^{\prime}\right) \leq \alpha\left(T^{\prime}\right)$.

(i) By replacing $X$ by $\operatorname{cl}(D(T))$, if necessary, we may assume $T$ is densely defined. Since $\beta(T)<\infty$, there exists a $p$ and $W$ as in Lemma 2 . For $n \geq p,\left(T^{\prime}+K_{n}^{\prime}\right) W^{\circ}$ is closed by preliminary remark I. Since $\alpha\left(T^{\prime}\right)=$ $\beta(T)<\infty,\left(T^{\prime}+K_{n}^{\prime}\right) Y^{\prime}=\left(T^{\prime}+K_{n}^{\prime}\right) W^{\circ}+K_{n}^{\prime} \pi\left(T^{\prime}\right)$ is closed; i.e., $T^{\prime}+K_{n}^{\prime}$ has a closed range and therefore $T+K_{n}$ has a closed range. Thus by what we have already shown,

$$
\beta\left(T+K_{n}\right)=\alpha\left(T^{\prime}+K_{n}^{\prime}\right) \leq \alpha\left(T^{\prime}\right)=\beta(T), \quad n \geq p .
$$

(ii) Suppose $\beta\left(T+K_{n}\right)=\beta(T)<\infty$ or equivalently $\alpha\left(T^{\prime}+K_{n}^{\prime}\right)=$ $\alpha\left(T^{\prime}\right), n \geq p$, with $p$ and $c$ chosen as in Lemma 2. Then $Y^{\prime}=r\left(T^{\prime}\right) \oplus$ $W^{\circ}=\Re\left(T^{\prime}+K_{n}^{\prime}\right) \oplus W^{\circ}$. Thus for $y^{\prime}=z_{n}^{\prime}+w_{n}^{\prime}, z_{n}^{\prime} \in \Re\left(T^{\prime}+K_{n}^{\prime}\right), w_{n}^{\prime} \in W^{\circ}$, we have 


$$
\left\|\left(T^{\prime}+K_{n}^{\prime}\right) y^{\prime}\right\|=\left\|\left(T^{\prime}+K_{n}^{\prime}\right) w_{n}^{\prime}\right\| \geq c\left\|w_{n}^{\prime}\right\| \geq c d\left(y^{\prime}, \Re\left(T^{\prime}+K_{n}^{\prime}\right)\right) .
$$

Hence $\gamma\left(T+K_{n}\right)=\gamma\left(T^{\prime}+K_{n}^{\prime}\right) \geq c, n \geq p$.

Theorem 3. Let $T$ be a Fredholm operator. Then $\gamma\left(T+K_{n}\right)$ is bounded away from zero for all sufficiently large $n$ if and only if $\alpha\left(T+K_{n}\right)$ $=\alpha(T)$ and $\beta\left(T+K_{n}\right)=\beta(T)$ for all sufficiently large $n$.

Proof. By replacing $X$ by $\operatorname{cl}(\mathcal{D}(T))$, if necessary, we may assume $T$ is densely defined. $Y=R(T) \oplus W, W$ finite dimensional. Suppose $\gamma\left(T+K_{n}\right) \geq c>0$ for all but a finite number of $n$ but that $\beta\left(T+K_{n}\right) \neq$ $\beta(T)$ for infinitely many $n$. Then by (i) of Theorem $2, \beta\left(T+K_{n}\right)<\beta(T)$ for infinitely many $n$. For simplicity, suppose $\beta\left(T+K_{n}\right)<\beta(T)$ and $\gamma\left(T+K_{n}\right) \geq c$ for $n \geq p$, where $p$ is chosen so that Lemma 2 holds. Thus there exists $y_{n} \in R\left(T+K_{n}\right) \cap W,\left\|y_{n}\right\|=1$. Since $\left\|y_{n}\right\|$ is bounded and $\gamma\left(T+K_{n}\right) \geq c>0$ it follows that there exists a bounded sequence $\left\{x_{n}\right\}$ such that $y_{n}=\left(T+K_{n}\right) x_{n}$. Now $\left\{y_{n}\right\}$ has a convergent subsequence since $W$ is finite dimensional; say $y_{n^{\prime}} \rightarrow y \in W$. Since $\left\{K_{n^{\prime}} x_{n^{\prime}}\right\}$ has a convergent subsequence, so does $\left\{T x_{n^{\prime}}\right\}$. Thus by preliminary remarks IV and II, $\left\{x_{n^{\prime}}\right\}$ has a convergent subsequence and $\left\{K_{n^{\prime}} x_{n^{\prime}}\right\}$ has subsequence $\left\{K_{n^{\prime \prime}} x_{n^{\prime \prime}}\right\}$ converging to zero. Hence $y=\lim y_{n^{\prime \prime}}=\lim T x_{n^{\prime \prime}} \in \mathfrak{R}(T)$, which shows that $y$ is in $R(T) \cap W=(0)$. This is impossible since $\|y\|=1$. The rest of the theorem follows from Theorem 1 .

Theorem 4. Let $T$ be a semi-Fredholm operator. There exists a $p$ such that for $n \geq p$,

(1) $T+K_{n}$ is semi-Fredholm,

(2) $\alpha\left(T+K_{n}\right) \leq \alpha(T)$,

(3) $\beta\left(T+K_{n}\right) \leq \beta(T)$,

(4) $\kappa\left(T+K_{n}\right)=\kappa(T)$.

Proof. The first three conclusions are contained in Theorems 1 and 2. There exists a $p$ such that for all $\lambda \in[0,1]$ and $n \geq p, T+\lambda K_{n}$ is semiFredholm. If this is not the case, there exists a subsequence $\left\{K_{n^{\prime}}\right\}$ and a sequence $\lambda_{n} \in[0,1]$ such that $T+\lambda_{n} K_{n^{\prime}}$ is not semi-Fredholm. This is impossible by Theorems 1 and 2 since $\lambda_{n} K_{n} \stackrel{c}{\rightarrow} 0$. Given $n \geq p$, define $\phi$ on $[0,1]$ with values in the set oxtended integers with the discrete topology by $\phi(\lambda)=\kappa\left(T+\lambda K_{n}\right)$. By [3, V.1.6], $\phi$ is continuous, and since 
$[0,1]$ is connected, $\phi$ is constant. In particular $\kappa(T)=\phi(0)=\phi(1)=$ $\kappa\left(T+K_{n}\right)$.

Remark. In Bull. Austral. Math. Soc. 8 (1973), 279-287, Lo proved the following theorem.

Let $T, T_{n}$ be bounded linear operators on $X, T$ compact and $\left\|T_{n}-T\right\|$ $\rightarrow 0$. Let $\mu \neq 0$ be an eigenvalue of $T$, and let $\mu_{n}$ be eigenvalues of $T_{n}$ such that $\mu_{n} \rightarrow \mu$. Then the following are equivalent:

(a) $\operatorname{dim} \Upsilon\left(\mu_{n}-T_{n}\right)=\operatorname{dim} \Re(\mu-T)$ eventually;

(b) for every $x$ in $\Re(\mu-T),\|x\|=1$, there is a sequence $\left\{x_{n}\right\}$ such that $x_{n} \in \pi\left(\mu_{n}-T_{n}\right)$ and $x_{n} \rightarrow x_{\text {. }}$

The above result is a very special case of Theorem 1. For consider $\mu_{n}-T_{n}=\mu-T+K_{n}$ where $K_{n}=\mu_{n}-\mu+T-T_{n}$. Then $\left\|K_{n}\right\| \rightarrow 0$ and therefore $K_{n} \stackrel{c}{\rightarrow} 0$. Since $T$ is compact, $\mu-T$ is a Fredholm operator. Assuming (a), we have, by Theorem 1 , the existence of a $c>0$ such that for all $n$ sufficiently large and $x \in \Re(\mu-T)$,

$$
\left\|K_{n} x\right\|=\left\|\left(\mu_{n}-T_{n}\right) x\right\| \geq c d\left(x, \Re\left(\mu_{n}-T_{n}\right)\right) .
$$

Since $K_{n} x \rightarrow 0$, (b) follows. On the other hand, if (b) holds, then Theorem 1 together with the basic lemma imply (a).

The proof shows that $T$ need not be compact but only that $\mu-T$ be semi-Fredholm with $a(\mu-T)<\infty$. Moreover, $\left\|T_{n}-T\right\| \rightarrow 0$ can be replaced by $T_{n}-T \stackrel{c}{\rightarrow} 0$. If we stipulate that $\mu-T$ be Fredholm, then to (a) in the above theorem we may add $\beta\left(\mu_{n}-T_{n}\right)=\beta(\mu-T)$ for all $n$ sufficiently large. This is a consequence of Theorem 3 .

\section{REFERENCES}

1. P. M. Anselone, Collectively compact operator approximation theory, Prentice-Hall, Englewood Cliffs, N. J., 1971.

2. I. C. Gohberg and M. G. Krein, The basic propositions on defect numbers, root numbers and indices of linear operators, Uspehi Mat. Nauk 12 (1957), no. 2 (74), 43-118; English transl., Amer. Math. Soc. Transl. (2) 13 (1960), 185-264. MR 20 \#3459; 22 \#3984.

3. S. Goldberg, Unbounded linear operators: Theory and applications, McGrawHill, New York, 1966. MR 34 \#580.

4. T. Kato, Perturbation theory for linear operators, Die Grundlehren der math. Wissenschaften, Band 132, Springer-Verlag, New York, 1966. MR 34 \#3324.

5. L. S. Rakovščik, The stability of the index and semistability of the defect numbers in compact approximation, Sibirsk. Mat. Ž. 13 (1972), 433-438 = Siberian Math. J. 13 (1972), 630-637.

DEPARTMENT OF MATHEMATICS, UNIVERSITY OF MARYLAND, COLLEGE PARK, MARYLAND 20742 